\title{
Estado de nutrición en hierro en una población de 4 a 14 años, urbano marginal, de Lima
}

\author{
Alicia Fernández ${ }^{1}$, Luzmila Troncoso ${ }^{1}$, Violeta Nolberto ${ }^{2}$
}

Resumen

Palabras clave

\begin{abstract}
Objetivo: Determinar el estado de nutrición en hierro en una población de 4 a 14 años. Diseño: Estudio descriptivo, observacional, transversal y prospectivo. Lugar: Facultad de Medicina, Universidad Nacional Mayor de San Marcos. Participantes: Niños, entre 4 y 14 años. Intervenciones: Se estudió 349 niños, 170 de sexo masculino y 179 de sexo femenino, entre 4 y 14 años de edad, del Centro de Salud San Genaro de Villa, Chorrillos, entre setiembre 2001 y agosto 2002. Principales medidas de resultados: Estado de nutrición en hierro. Resultados: El 68,8\% (240) no presentó alteración en el estado de nutrición en hierro(ENH), frente a 31,2\% (109) que sí lo presentó. De los 109 niños con alteración en el ENH, 68,8\% (75) clasificó en el estadio I de depleción latente (ferritina $<20 \mathrm{ng} / \mathrm{mL}$ ), 22,9\% (25), en el estadio II de ferropenia (hierro sérico $<60 \mathrm{ug} / \mathrm{dL}$ y capacidad total de fijación del hierro $>400 \mathrm{ug} / \mathrm{dL}$ ), y8,3\% (9), en el estadio III de anemia ferropénica (hemoglobina $<11,5$ g/100 mL para menores de 11 años $<<12$ g/100 $m L$ para 12 a 14 años, hematocrito $<34$ para menores de 11 años $y<36 \%$ para 12 a 14 años, volumen corpuscular medio <80 fl, y hemoglobina corpuscular media <27pg). Conclusiones: El alto porcentaje de niños con alteración en el estado de nutrición en hierro, estadio I, identifica a una población en riesgo de anemia ferropénica. Se recomienda que los estudios de deficiencia en hierro se restrinjan a niños en estadio I, con el fin de establecer políticas de participación ciudadana y establecer alianzas sectoriales y extrasectoriales (sector educación), para la promoción de la nutrición infantil.
\end{abstract}

Estado nutricional; anemia ferropénica; trastornos de la nutrición del niño.
Iron nutrition status in 4 to 14 year-old children living in a marginal urban section in Lima

Abstract

Objective: To determine the iron nutrition status in 4 to 14 yearold children. Design: Descriptive, observational, transversal and prospective study. Setting: Faculty of Medicine, Universidad Nacional Mayor de San Marcos. Participants: Four to fourteen year-old children. Interventions: We studied 349 children 4 to 14 year-old, 170 males and 179 females, at San Genaro Health Center, Chorrillos, from September 2001 through August 2002. Main outcome measures: Iron nutritional status. Results: From these children, 68,8\% (240) did not present alteration in iron nutrition status (INS), compared with $31,2 \%$ who presented this alteration. From the 109 children with iron nutrition status alteration, 68,8\% (75) were stage I or latent depletion (ferritine $<20 \mathrm{ng} / \mathrm{mL}$ ); 22,9\% (25) were stage II or iron deficient (serum iron $<60 \mathrm{ug} / \mathrm{dL}$ and iron total capacity offixation $>400 \mathrm{ug} / \mathrm{dL}$ ), and 8,3\% (9) were in stage III with iron deficient anemia

1 Facultad de Medicina, UNMSM. Lima, Perú.

2 Facultad de Matemáticas, UNMSM. Lima, Perú. (hemoglobin $<11,5 \mathrm{~g} / 100 \mathrm{~mL}$ for children less than 11 year-old and $<12 \mathrm{~g} / 100 \mathrm{~mL}$ for children 12 to 14 year-old, hematocrit $<34 \%$ for children less than 11 year-old and $<36 \%$ for children 12 to 14 year-old, median corpuscupar volume $<80$ fl and median corpuscular hemoglobin $<27$ pg). Conclusions: The high percentages of children with alteration of the iron nutrition status stage I identifies a population with iron deficiency anemia risk. We recommend that iron deficiency studies be applied only to children in stage I, in order to institute citizen participation public policies and establish alliances with other sectors (education), for child nutrition promotion.

Key words: Nutritional status; anemia, iron-deficiency; child nutrition disorders.

\section{INTRODUCCIÓN}

Las carencias de micronutrientes, es decir deficiencias de vitaminas y minerales, constituyen la forma de malnutrición más generalizada del mundo $\left({ }^{1}\right)$. Más de dos mil 
millones de personas sufren en el mundo de distintos tipos de carencia de micronutrientes. De acuerdo con estimaciones de la OMS/ UNICEF/UNU, la deficiencia de hierro es la deficiencia nutricional más ampliamente extendida en el mundo. Pese a que el problema es más grave en los países de economía agrícola, las naciones industrializadas no escapan a este mal; mientras en éstas $11 \%$ de sus habitantes presenta anemia por deficiencia de hierro, en aquellos, el padecimiento afecta a cerca de la tercera parte de la población $\left({ }^{2}\right)$. La prevalencia de la deficiencia subclínica de hierro es al menos el doble que la de la anemia $\left({ }^{3}\right)$. Se puede estimar que más de la mitad de la población de la región de América Latina y el Caribe actualmente presenta deficiencia de hierro $\left({ }^{4}\right)$.

De los elementos traza, el hierro ha recibido la mayor atención en nutrición infantil. Sin embargo, a diferencia de otros problemas de salud, tales como el cáncer o las enfermedades cardiovasculares, la deficiencia de hierro no aparece en las estadísticas de mortalidad. La falta de datos epidemiológicos se debe a que sus manifestaciones son generalmente tan ligeras e insignificantes que rara vez motivan una consulta con el pediatra.

La escasez de hierro no se distribuye de manera homogénea a lo largo de la población. Los grupos más vulnerables son las mujeres en edad reproductiva -en especial las embarazadas-, los niños y los adolescentes. Se debe a que en dichas etapas de la vida las demandas de hierro se incrementan en forma notoria y en ocasiones la dieta no es suficiente para cubrirlas. La anemia tiene serias consecuencias en la calidad de vida. Estas repercusiones están ligadas a la disminución de la eficiencia de transporte de oxígeno a los diferentes tejidos del cuerpo, que es una función prioritaria del organismo $\left({ }^{5}\right)$. Durante la etapa de crecimiento y desarrollo, el impacto de la deficiencia de hierro y la anemia acarrean graves consecuencias, entre otras áreas, en la psicomotora e intelectual que, en ocasiones solo se evidencian a largo plazo $\left({ }^{6,7}\right)$. La carencia de hierro afecta principalmente a la inmunidad celular, función intestinal, crecimiento y rendimiento físico; también a la conducta, metabolismo de las catecolaminas y termogénesis. En personas con deficiencia de hierro se ha identificado varias anomalías inmunológicas, entre ellas, un menor porcentaje de linfocitos $\mathrm{T}$, defectos en la respuesta inmunitaria mediada por células, empeoramiento de la transformación de los linfocitos, menos reacciones cutáneas positivas a los antígenos comunes, y una disminución de la mieloperoxidasa de los granulocitos, con menor capacidad de destrucción microbiana $\left({ }^{8,9}\right)$.

A nivel del sistema nervioso, los niños con deficiencia de hierro presentan en mayor o menor medida irritabilidad, apatía, alteraciones del lenguaje, disminución de la atención y/o concentración y, lo más importante, produce afectación del desarrollo psicomotor y coeficiente intelectual $\left({ }^{10}\right)$.

El hierro presente en el organismo puede ser dividido en dos componentes principales: el hierro funcional y el hierro almacenado. El componente funcional consiste en el hierro contenido en la hemoglobina circulante, con cantidades menores en los tejidos del organismo, en la mioglobina y en diversas enzimas heme y no heme. Aunque la carencia de hierro en los tejidos es más difícil de definir, desde el punto de vista de la salud, es probablemente tan importante como la carencia más obvia de hemoglobina, que provoca anemia $\left({ }^{11}\right)$. Es difícil medir la deficiencia de hierro en los tejidos, pero si el aporte de hierro a la médula eritroide es suficiente (medido en el laboratorio mediante la saturación normal de transferrina y la protoporfirina en los glóbulos rojos), las necesidades tisulares de hierro están plenamente cubiertas. 
El hierro almacenado sirve como reserva para reemplazar las pérdidas del componente funcional. El organismo contiene depósitos de hierro en forma de ferritina y de hemosiderina en el hígado, el bazo y la médula ósea. En un hombre adulto sin deficiencia de hierro, el hierro almacenado asciende a una cuarta parte del hierro total del organismo. En cambio, cuando las necesidades fisiológicas de hierro son altas, como en los niños y mujeres embarazadas, los depósitos de hierro son a menudo escasos o nulos.

El estado nutricional en hierro se puede conocer mediante tres tipos de evaluación: la clínica, la dietética y la bioquímica; las dos primeras proporcionan pautas para sospechar la deficiencia, mientras que solo con la tercera se puede establecer claramente un diagnóstico. Para valorar el estado en hierro existe una serie de determinaciones bioquímicas que estiman diferentes estadios de la carencia $\left({ }^{12}\right)$.

- Estadio I, depleción de los depósitos de hierro del organismo. El marcador bioquímico relacionado directamente con el tamaño de estos depósitos es la ferritina sérica (FS).

- Estadio II, deficiencia. En este estadio pueden aparecer alteradas las siguientes determinaciones bioquímicas: la FS, el hierro sérico (HS), la transferrina sérica (TS), la capacidad total de fijación del hierro (CTFH), el coeficiente de saturación de la transferrina $(\mathrm{CST}=\mathrm{HS} / \mathrm{CTFH})$ y la protoporfirina eritrocitaria (PE) $\left({ }^{13,14}\right)$.

- Estadio III, anemia ferropénica. Acontece cuando ante una ferropenia se afectan los niveles de la hemoglobina ( $\mathrm{Hb})$ y el hematocrito (Hto).

La mayoría de trabajos de investigación se centran en los valores de $\mathrm{Hb}$ y Hto (estadio III). El objetivo del presente trabajo es determinar los estadios previos, a decir, la población en riesgo de presentar anemia ferropénica.

\section{MATERIALES Y MÉTODOS}

Se trata de un estudio descriptivo, observacional, transversal y prospectivo.

Se estudió 349 niños de ambos sexos, entre 4 a 14 años de edad, (agrupados por edades de 4 a 7 años, de 8 a10 años y de 11 a 14 años), que acudieron a la campaña de despistaje de anemia al Centro de Salud San Genaro de Villa, Chorrillos. Las condiciones geográficas propias de la zona, son suelo arenoso, clima subtropical y con 26 asentamientos humanos, el $50 \%$ de la población posee viviendas de material rústico. El abastecimiento de agua se da a través de dos modalidades, por red pública y camiones cisterna $\left({ }^{15}\right)$.

Se registró los datos en una historia clínica para cada niño, luego se obtuvo muestras de sangre venosa, en ayunas, para los exámenes bioquímicos. Todas las determinaciones fueron realizadas el mismo día de la toma de la muestra y procesadas en el Laboratorio Oswaldo Hercelles, del Hospital Nacional Dos de Mayo.

Con relación a la medición de las variables, se siguió los siguientes procedimientos:

Estadio I, depleción. A través de la determinación de la ferritina sérica, utilizando el método de inmunoensayo de electroquimioluminiscencia (ECLIA), por analizadores Elecsys 2010, de Roche., utilizando como punto de corte $<20 \mathrm{ng} / \mathrm{mL}$.

Estadio II, ferropenia o deficiencia. Por las determinaciones de hierro sérico, con valor de corte de $<60 \mathrm{ug} / \mathrm{dL}, \mathrm{CTFH}$ con valor de corte $=0>400 \mathrm{ug} / \mathrm{dL}$ y la saturación de transferrina, con valores de $<12 \%$ (1 a 4 años), <14\% (5 a 10 años) y $<16 \%$ $\left(>10\right.$ años) $\left({ }^{13}\right)$. Se realizó por determina- 
ción colorimétrica, utilizando reactivos Valtek (Chile).

Estadio III, anemia ferropénica. Por la determinación de $\mathrm{Hb}$. Por el método de cianometahemoglobina, expresándose la concentración en g/100 mL. Considerando los puntos de corte para los 4 años $<11 \mathrm{~g} /$ $100 \mathrm{~mL}$, de 5 a 11 años $<11,5 \mathrm{~g} / 100 \mathrm{~mL}$ y de 12 a 14 años $<12 \mathrm{~g} / 100 \mathrm{~mL}$ (WHO, UNICEF, 1998). Los valores de hematocrito eran $<33,<34$ y $<36 \%$, respectivamente (efectuado por centrifugación de la sangre con oxalato y lectura directa).

Los índices celulares VCM, fueron determinados por medio de Cell-dyn (Abbott), que considera valor de corte $<80 \mathrm{fl}$, indicando microcitosis, y $\mathrm{HbCM}<27 \mathrm{pg}$, que indica hipocromía.

\section{RESULTADOS}

Los niños fueron 170 del sexo masculino y 179 del femenino, 131 de 4 a 7 años, 118 entre 8 y 10 años y 100 entre 11 y 14 años. Figura 1.

Se evaluó el estado nutricional en hierro por medio de las siguientes variables: Figura 2 .

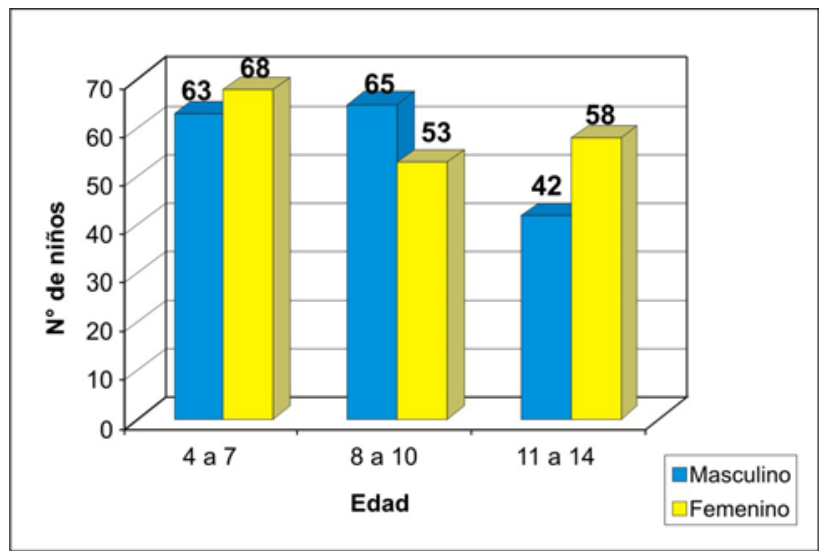

Figura 1. Distribución de niños por edad y sexo.
1. Constantes corpusculares.- Hemoglobina corpuscular media ( $\mathrm{HbCM})$, con promedio de $28,25 \mathrm{pg}, 15,8 \%$ por debajo de los parámetros establecidos, hipocromía. Volumen corpuscular medio (VCM) con promedio de $82,09 \mathrm{fl} ; 25,8 \%$ presentó microcitosis.

2. Hemoglobina $(\mathrm{Hb})$.- Con un promedio global de $12,5 \mathrm{~g} / 100 \mathrm{~mL}, 11,7 \%$ de niños por debajo de los parámetros establecidos, y hematocrito (Hto) con 15,2\% de niños por debajo de los parámetros establecidos.

3. Porcentaje de saturación de transferrina, (Sat) promedio global de $19,9 \%$ y se encontró alterado en $44,1 \%$ de niños, con mayor frecuencia en el sexo femenino.

4. Capacidad total de fijación de hierro (CTFH), con promedio global de 312,18 ug/dL y estuvo alterada en $14,3 \%$, considerando como valor anormal el mayor de $400 \mathrm{ug} / \mathrm{dL}$,

5. Hierro sérico (HS).- El promedio global fue $59,88 \mathrm{ug} / \mathrm{dL} ; 57,3 \%$ de los niños presentó hierro sérico por debajo de 60 ug/ d L.

6. Ferritina sérica (FS).- Con promedio global de $34,64 \mathrm{ng} / \mathrm{mL}$. Con corte de 20 $\mathrm{ng} / \mathrm{mL}$-depleción latente-, $25,8 \%$, de niños estaba afectados, con mayor frecuencia en el sexo femenino.

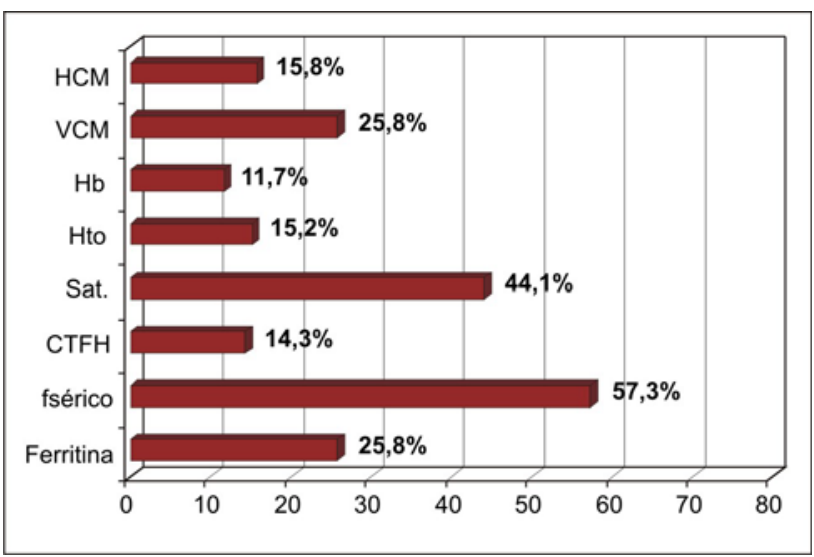

Figura 2. Porcentaje de niños por debajo de los puntos de corte establecidos. 
Tabla 1. Distribución de niños por estado nutricional en hierro según estadios.

\begin{tabular}{lrrrrrr}
\hline \multirow{2}{*}{ Estadio } & \multirow{2}{*}{ Total } & \multicolumn{3}{c}{ Estado nutricional del hierro } \\
\cline { 3 - 4 } & & \multicolumn{2}{c}{ Alterado } & & \multicolumn{2}{c}{ Normal } \\
\cline { 3 - 4 } \cline { 6 - 7 } & & $\mathrm{n}$ & $\%$ & & $\mathrm{n}$ & $\%$ \\
\hline \multirow{2}{*}{ Normal } & 240 & 0 & 0 & & 240 & 100 \\
Estadio I & 75 & 75 & 68,8 & & 0 \\
Estadio II & 25 & 25 & 22,9 & & 0 & 0 \\
Estadio III & 9 & 9 & 8,3 & & 0 & 0 \\
Total & 349 & 109 & 100 & & 240 & 100 \\
\hline
\end{tabular}

Al clasificar los niños por estadios, se observa que $68,8 \%$ (240) se encontraba con ENH normal frente a $31,2 \%$ (109) que presentaba alteración en el ENH. Ver Tabla 1, Figuras 3 y 4.

De los 109 niños con ENH alterado, en el estadio I (depleción) $(68,8 \%)$ existió mayor frecuencia del sexo femenino y edad entre 11 y 14 años de edad. En el estadio II (deficiencia) $(22,9 \%)$, hubo también mayor frecuencia del sexo femenino, pero la edad entre 8 y 10 años; en el estadio III (anemia ferropénica) $(8,3 \%)$, la frecuencia se inclinó hacia el sexo masculino y entre 4 y 7 años de edad. Ver Tabla 2.

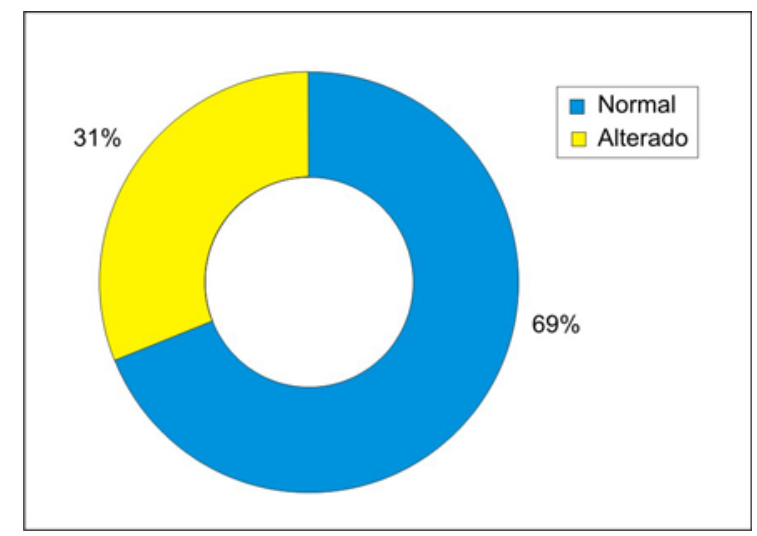

Figura 3. Niños según estado nutricional en hierro.

\section{DISCUSIÓN}

Al medir los parámetros hematológicos y bioquímicos, $57,3 \%$ (200) de los niños presentó hierro sérico bajo (HS). Esta cifra es muy elevada, si se compara con estudios similares realizados en adolescentes costarricenses, donde solamente $3,4 \%$ mostró niveles bajos de hierro sérico $\left({ }^{16}\right)$.

Al clasificar el ENH por estadios, se observa que $68,8 \%$ (240) de los niños tenía ENH adecuado, frente a $31,2 \%$ (109) de los niños que presentó alteración en el ENH.

De los 109 niños con alteración en el ENH, $8,3 \%$ (9) presentó anemia ferropénica (estadio III), con mayor frecuencia en el sexo masculino y entre $\operatorname{los} 4$ y 7 años de edad (Tabla 2), cifras inferiores a las descritas por Pajuelo (1992), en 2678 niños de 3 a 71 meses de edad, que revelaron tasas globales de anemia de $42 \%$, tendiendo a declinar conforme aumentaba la edad. También, estudios de la Dra. Zavaleta (1993), con una prevalencia de anemia de $64 \%$ en niños menores de 6 años $\left({ }^{17}\right)$, y PRISMA (1995), en 280 niños de 1 a 4 años, de Lima, donde las tasas de anemia fueron $12,9 \%$ en el grupo socioeconómico alto, $26,7 \%$ en el grupo socioeconómico bajo y $27 \%$ en las barriadas; finalmente, el estu-

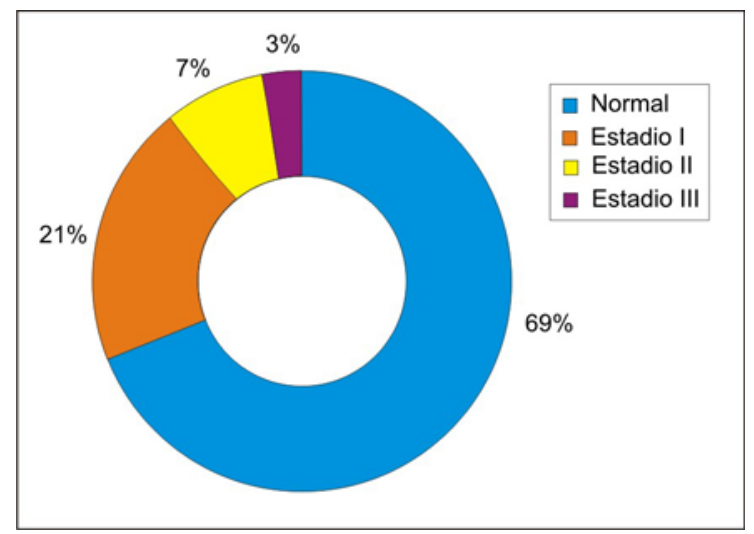

Figura 4. Niños según estadios del estado de nutrición en hierro. 
Tabla 2. Distribución de niños según estadios, edad y sexo.

\begin{tabular}{|c|c|c|c|c|c|c|c|c|c|}
\hline \multirow{3}{*}{ Edad } & \multirow{3}{*}{ Sexo } & \multicolumn{6}{|c|}{ Estadio } & & \\
\hline & & \multicolumn{2}{|c|}{ Estadio I } & \multicolumn{2}{|c|}{ Estadio II } & \multicolumn{2}{|c|}{ Estadio III } & \multicolumn{2}{|c|}{ Total * } \\
\hline & & $n$ & $\%$ & $\mathrm{n}$ & $\%$ & $\mathrm{n}$ & $\%$ & $\mathrm{n}$ & $\%$ \\
\hline \multirow[t]{3}{*}{$4-7$} & Masculino & 17 & 30,91 & 6 & 10,91 & 4 & 7,27 & & \\
\hline & Femenino & 22 & 40,0 & 6 & 10,91 & 0 & 0,00 & & \\
\hline & Total & 39 & 70,91 & 12 & 21,82 & 4 & 7,27 & 55 & 50,46 \\
\hline \multirow[t]{3}{*}{$8-10$} & Masculino & 9 & 33,33 & 3 & 11,11 & 2 & 7,41 & & \\
\hline & Femenino & 7 & 25,93 & 6 & 22,22 & 0 & 0,00 & & \\
\hline & Total & 16 & 59,26 & 9 & 33,33 & 2 & 7,41 & 27 & 24,77 \\
\hline \multirow[t]{3}{*}{$11-14$} & Masculino & 4 & 14,81 & 1 & 3,70 & 2 & 7,41 & & \\
\hline & Femenino & 16 & 59,26 & 3 & 11,11 & 1 & 3,70 & & \\
\hline & Total & 20 & 74,07 & 4 & 14,81 & 3 & 11,11 & 27 & 24,77 \\
\hline Total $^{\dagger}$ & & 75 & 68,81 & 25 & 22,94 & 9 & 8,26 & 109 & 100,00 \\
\hline
\end{tabular}

* Por estadios

+ Por grupos de edad

dio de Campos y col. (1997), en 1861 niños de 3 a 11 años, en Lima, donde la tasa de anemia era $20 \%$, utilizando todos la variable hemoglobina $(\mathrm{Hb})\left({ }^{18}\right)$. Según valores de la OPS/OMS, la incidencia de anemia en menores de 5 años fue $50 \%$ a $78 \%$, entre los 12 y 15 años de edad $\left({ }^{19}\right)$. En América Latina, estudios en Buenos Aires y Tierra del Fuego realizados por CESNI. (1992, 1995) dan una prevalencia de $12,8 \%$ y $10 \%$ para la población escolar; en países andinos, esta cifra se eleva hasta $30 \%\left({ }^{20}\right)$.

E1 22,9\% (25) de los niños presentó ferropenia (estadio II), con mayor frecuencia en el sexo femenino y entre 8 y 10 años de edad. En la anemia ferropénica, la sideremia suele hallarse disminuida $(<60$ $\mathrm{ug} / \mathrm{dL}$ ) y se acompaña de un característico aumento de la CTFH y de una disminución del índice de saturación de la transferrina (\% sat.). La valoración conjunta de estas tres magnitudes es de gran utilidad, no solo para conocer la etapa en que se halla el desarrollo de la anemia ferropénica, sino también en el diagnóstico diferencial con la anemia por enfermedad crónica (APEC), en la que la disminución de la sideremia es muy característica, pero CTFH suele hallarse normal $\left({ }^{12}\right)$. En estudios realizados en población escolar española, se ha observado una menor prevalencia de ferropenia $1,6 \%$ a $2,6 \%\left({ }^{13}\right)$-, no encontrando cifras comparables en la población peruana.

El $68,8 \%$ (75) de los niños presentó depleción (estadio I), con mayor frecuencia en el sexo femenino y entre los 11 y 14 años de edad, que van hacia los estadios evolutivos propios de la enfermedad sino se corrige los factores desencadenantes. Estas cifras son superiores a las descritas en el estudio de PRISMA (1995), en 280 niños de 1 a 4 años, donde se midió los niveles de ferritina sérica $(<12 \mathrm{ug} / \mathrm{dL})$ en $46 \%$ de los niños.

En Argentina, Calvo y colaboradores (1990) y CESNI (1995) dan cifras de $56 \%$ y $16 \%$, respectivamente, con nivel sérico de ferritina de $<12 \mathrm{ug} / \mathrm{dL}$. Colombia, según el Ministerio de Salud y el Instituto Nacional de Salud (1995), señala prevalencia de $18 \%$, para la ferritina sérica $<12$ ug/dL, y de $42 \%$ en niños con ferritina sérica $<24 \mathrm{ug} / \mathrm{dL}$. 
En Méjico, Rosado y col. (1994), y Costa Rica, Ministerio de Salud (1996), señalan cifras de prevalencia de $52 \%$ y $56,3 \%$, respectivamente, con ferritina sérica $<12$ $\mathrm{ug} / \mathrm{dL}\left({ }^{14}\right)$. Estudios en población española muestran que, a pesar de una menor prevalencia de ferropenia y de anemia ferropénica, los niños de 4 a 16 años presentan depósitos nulos de hierro en $20 \%$ $\left({ }^{21}\right)$.

Como conclusiones, podemos decir lo siguiente. En los parámetros bioquímicos y hematológicos, 57,3\% (200) de los niños presentó hierro sérico (HS) bajo, pero solo 31,2\% (109) mostró alteración en el ENH, distribuidos en sus 3 estadios. Existen otras variables que modifican el ENH en esta población, como la biodisponibilidad de hierro de la dieta y la presencia de enfermedades crónicas, que es necesario controlar para poder prevenir el desarrollo de anemia ferropénica en esta población infantil.

Se recomienda determinar ferritina sérica (estadio I), como medida para prevenir los desórdenes en el metabolismo del hierro en la población infantil, reforzar los programas de educación alimentaria dirigidos a madres y organizaciones comunales; donde se oriente sobre la importancia del consumo de hierro, dar mayor atención a los programas de salud infantil de las áreas urbano marginales, aumentar el control de las infecciones en los niños y establecer alianzas sectoriales y extrasectoriales para la promoción de la salud infantil.

\section{REFERENCIAS BIBLIOGRÁFICAS}

1. Lynch S. Food iron absorption and its importance for the design of food fotification strategies. Nutrition reviews. 2002;60(7):S3-6.

2. FAO/OMS. Nutrición y desarrollo. Conferencia internacional sobre nutrición. Roma: FAO/OMS; 1992.

3. Dallman PR, Simes MA, Sketel A. Iron deficiency in infancy and childhood. Am J Nutr. 1980;33:86-118.
4. Gueri M. Deficiencias de micronutrientes en Las Américas OPS. Bolletin de la Oficina Panamericana. 1994;117(6): 47782.

5. Yip R. Iron deficiency: contemporary scientific issues and international programmatic approaches. J Nutr. 1994;124(8 Suppl 1):1479S-1490S. Review.

6. Lozoff MD, Jiménez E. Long term developmental outcome of infants with iron deficiency. JAMA. 1991;325(10):68794.

7. Cortez R. Salud, equidad y pobreza en el Perú: teoría y nuevas evidencias. Lima: Centro de Investigación de la Universidad del Pacífico; 2002.

8. Ruíz M. Epidemología, etiología y factores de riesgo de la IRA. 1ra Edición. Lima; 1994. p. 66-9.

9. Vera J. Hierro, infección y nutrición. Asociación Colombiana de Nutrición Clínica. 1996;3(8):872-5.

10. Walter T. Impact of iron deficiency on cognition in infancy and childhood. New York: Nestle; 1992. p.30.

11. Dallman, Peter R. Hierro. Conocimientos actuales sobre nutrición. 6ta Edición. Washington: OPS; 1991. p. 277-88.

12. Sans J. Hematología clínica. 3ra edición. Madrid: MosbyDogma Libros SA; 1996.

13. Casanueva E. Nutriología Médica. México DF: Ed. Panamericana; 2001.

14. Paiva A, Rondó P. Parámetros para avaliacao do estado nutricional de ferro. Revista de Saude Pública. 2000;34.

15. Análisis de la Situación de Salud. Centro de Salud. San Genaro de Villa, 2001.

16. Muñoz L. Perfil férrico de adolescentes urbanos costarricenses. Rev Cost Cienc Méd. 1996;17(2):27-33.

17. Zavaleta N. Las Américas de hierro. Instituto de Investigación en Nutrición, 1998.

18. Mora J, Mora OL. Deficiencias de micronutrientes en América Latina y el Caribe. Anemia ferropriva. OPS/OMS, USAID, Roche, OMNI, 1997.

19. La Salud en las Américas. Perú. Enfermedades Nutricionales y del Metabolismo. OPS/OMS Ed. 2002;2:504.

20. Anemia por deficiencia de hierro en la región andina. Definición y estrategias de intervención Actas del SeminarioTaller UMSA-SNS-IBBA-ORSTOM. 26-29 de marzo 1996. La Paz, Bolivia.

21. Arija V, Fernández J. Carencia de hierro y anemia ferropénica en la población española. Medicina Clínica. 1997;109(11):425-30.

Manuscrito recibido el 12 de julio de 2007 y aceptado para publicación el 28 de sgosto de 2007.

Correspondencia:

Alicia Fernández Giusti

Facultad de Medicina, UNMSM.

Av. Grau 750. Lima 1, Perú.

Correo-e: aliciafer76@hotmail.com 